\title{
Enhanced read performance for phase change memory using a reference column
}

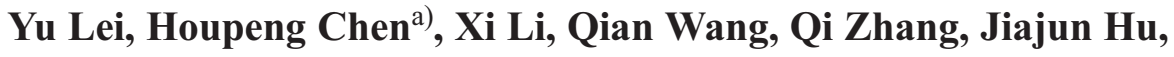 \\ Xiaoyun Li, Zhen Tian, and Zhitang Song \\ State Key Laboratory of Functional Materials for Informatics, Shanghai Institute of \\ Micro-system and Information Technology, Chinese Academy of Sciences, \\ Shanghai 200050, China
}

a)chp6468@mail.sim.ac.cn

\begin{abstract}
A reference column is employed to improve the read performance of phase change memory (PCM). In this way, a changeable reference current replaces the constant one; both the reference cell and the selected cell have the same bit line (BL) parasitic parameters and read transmission gate parasitic parameters in the read operation. Simulated in a $40 \mathrm{~nm}$ CMOS process, read access time of 4-Mb PCM is $30.65 \mathrm{~ns}$ with $190.9 \mathrm{~ns}$ improvement. Monte Carlo simulations show a $80.5 \mathrm{~ns}$ worst read access time compared to the conventional $1.58 \mu \mathrm{s}$.
\end{abstract}

Keywords: phase change memory, read access time, reference current, PCRAM, sense amplifier

Classification: Integrated circuits

\section{References}

[1] P. Junsangsri and F. Lombardi: "A new comprehensive model of a phase change memory (PCM) cell," IEEE Trans. Nanotechnol. 13 (2014) 1213 (DOI: 10.1109/TNANO.2014.2353992).

[2] D. H. Jin, et al.: “A $15 \mu \mathrm{m}$-Pitch, 8.7-ENOB, 13-Mcells/sec logarithmic readout circuit for multi-level cell phase change memory," IEEE J. Solid-State Circuits 50 (2015) 2431 (DOI: 10.1109/JSSC.2015.2453236).

[3] X. Li, et al:: "An SPICE model for PCM based on Arrhenius equation," Chin. Phys. Lett. 26 (2009) 128501 (DOI: 10.1088/0256-307X/26/12/128501).

[4] Y. Choi, et al.: "A $20 \mathrm{~nm} 1.8 \mathrm{~V} 8 \mathrm{~Gb}$ PRAM with $40 \mathrm{MB} / \mathrm{s}$ program bandwidth," ISSCC Dig. Tech. Papers (2012) 46 (DOI: 10.1109/ISSCC. 2012.6176872).

[5] D. H. Woo, et al.: "Pragmatic integration of an SRAM row cache in heterogeneous 3-D DRAM architecture using TSV," IEEE Trans. Very Large Scale Integr. (VLSI) Syst. 21 (2013) 1 (DOI: 10.1109/TVLSI.2011.2176761).

[6] Q. Wang, et al:: "Methods to speed up read operation in a 64 Mbit phase change memory chip,” IEICE Electron. Express 12 (2015) 20150792 (DOI: 10. 1587/elex.12.20150792).

[7] S. Hanzawa, et al: " A $512 \mathrm{kB}$ embedded phase change memory with $416 \mathrm{kB} / \mathrm{s}$ write throughput at $100 \mu$ A cell write current," ISSCC Dig. Tech. Papers (2007) 474 (DOI: 10.1109/ISSCC.2007.373500).

[8] K. Tsuchida, et al.: "A 64 Mbit MRAM with clamped-reference and adequatereference schemes," ISSCC Dig. Tech. Papers (2010) 258 (DOI: 10.1109/ 
ISSCC.2010.5433948).

[9] T. Andre, et al:: "A 4-Mb 0.18- $\mu \mathrm{m}$ 1T1MTJ toggle MRAM with balanced three input sensing scheme and locally mirrored unidirectional write drivers," IEEE J. Solid-State Circuits 40 (2005) 301 (DOI: 10.1109/JSSC.2004.837962).

[10] M. Chang, et al:: " 19.4 embedded $1 \mathrm{Mb}$ ReRAM in $28 \mathrm{~nm}$ CMOS with 0.27-to$1 \mathrm{~V}$ read using swing-sample-and-couple sense amplifier and self-boost-writetermination scheme,” ISSCC Dig. Tech. Papers (2014) 105 (DOI: 10.1109/ ISSCC.2014.6757457).

[11] H. Lung, et al.: "A double-data-rate 2 (DDR2) interface phase-change memory with $533 \mathrm{MB} / \mathrm{s}$ read -write data rate and $37.5 \mathrm{~ns}$ access latency for memorytype storage class memory applications," IEEE International Memory Workshop (2016) (DOI: 10.1109/IMW.2016.7493563).

[12] G. De Sandre, et al.: "A $4 \mathrm{Mb}$ LV MOS-selected embedded phase change memory in $90 \mathrm{~nm}$ standard CMOS technology," IEEE J. Solid-State Circuits 46 (2011) 52 (DOI: 10.1109/JSSC.2010.2084491).

[13] X. Fan, et al.: "Optimization of periphery circuits in a 1 K-bit PCRAM chip for highly reliable write and read operations," IEICE Electron. Express 11 (2014) 20141071 (DOI: 10.1587/elex.11.20141071).

\section{Introduction}

Phase change memory (PCM) has been attracting great interest as one of the most promising candidates for the next-generation nonvolatile memory devices [1]. The storage mechanism of PCM is based on reversible phase change transitions between a high resistance amorphous state (reset state for data "1") and a low resistance crystalline state (set state for data " 0 ") of a chalcogenide alloy $\left(\mathrm{Ge}_{2} \mathrm{Sb}_{2} \mathrm{Te}_{5}\right.$ or GST) [2, 3]. As a continuous growth in scalability, parasitic parameters in the array have been a major obstacle in reducing the read access time [4]. This limit of parasitic parameters has been discussed a lot in various kinds of memories [5], while that is not specific in PCM. In this paper, we will discuss a method for improving the read performance, which has been applied to the development of 4-Mb standalone PCM with $40 \mathrm{~nm}$ CMOS technology.

\section{Conventional read circuit analysis}

Fig. 1(a) shows schematic of the read circuit of PCM which is a fully differential sense amplifier (SA). The red zone is the selected 1T1R cell, which comprises a GST element $\mathrm{R}_{\mathrm{GST}}$ and a selection transistor NM0. The $1 \mathrm{~T} 1 \mathrm{R}$ cell is selected by a word line (WL) and a bit line (BL) from numbers of cells in the array. The read current $\mathrm{I}_{\text {read }}$ and the reference current $\mathrm{I}_{\text {ref }}$ are generated by $V_{\text {clamp }}$ and a reference circuit, respectively. A $\sim 100 \mathrm{mV} V_{R B L}$ is low enough to avoid the additional set operation. In the SA, PM2 and NM4 copy $I_{\text {read }}$, PM3 and NM3 copy $I_{\text {ref }}$. When reading a set cell, $I_{\text {read }}>I_{\text {ref }}$. So PM2 copies a larger current than NM3 does, $V_{1}$ will rise to near VDD; also NM4 copies a larger current than PM3 does, $V_{2}$ will drop to near $0 \mathrm{~V}$; DO of the SR latch will output VDD or "1".

When we consider the read operation, the effect of parasitic parameters in the 
in Fig. 1(b), bit line parasitic parameters include parasitic capacitors of the selection transistors. Read transmission gate parasitic parameters include parasitic capacitors and resistors of read transmission gates. There are $n 1 \mathrm{~T} 1 \mathrm{R}$ cells in one Local Bit Line (LBL) and $m$ LBLs share one SA. Bit line parasitic parameters are related to $n$; read transmission gate parasitic parameters are related to $m$. In the read operation, SA has to charge parasitic capacitors in the array at first.

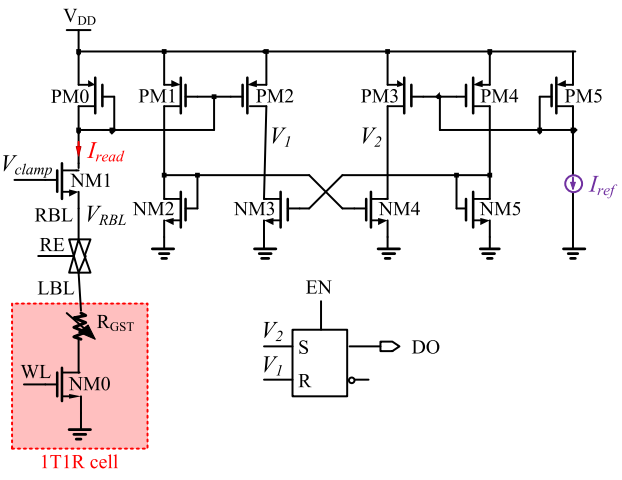

(a)

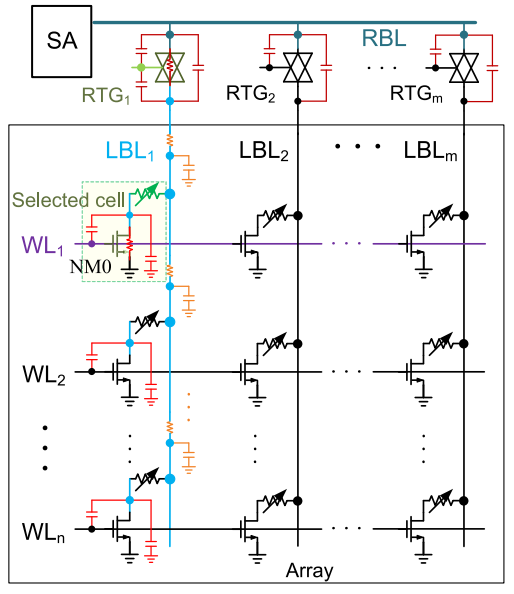

(b)

Fig. 1. (a) Schematic of the conventional read circuit. (b) Parasitic elements in the read operation: parasitic capacitors and resistors of wires (in orange); parasitic capacitors and resistors of selection transistors (in red); parasitic capacitors and resistors of read transmission gates (in ruby).

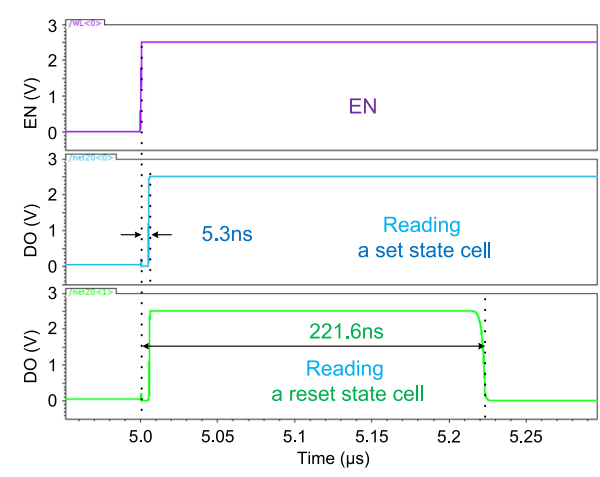

(a)

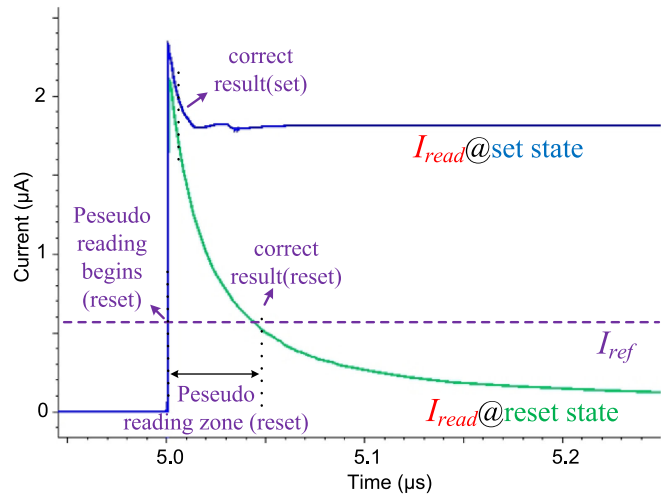

(b)

Fig. 2. (a) Simulation results of the conventional read access time. (b) $I_{\text {read }}$ and $I_{\text {ref }}$ versus time.

In this work, the GST resistance is modeled base on the conventional GST element used in a $40 \mathrm{~nm}$ PCM device [6]. The set resistance distribution is from a few $\mathrm{K} \Omega$ to $100 \mathrm{~K} \Omega$ and the reset resistance distribution is from $1 \mathrm{M} \Omega$ to dozens of $\mathrm{M} \Omega$.

The 64-Kb unit block of 4-Mb PCM contains 1024 X-selectors, 64 Y-selectors and one SA. Large numbers of X-selectors and Y-selectors result in considerable parasitic capacitors and resistors on BL. More parasitic elements result in a higher 


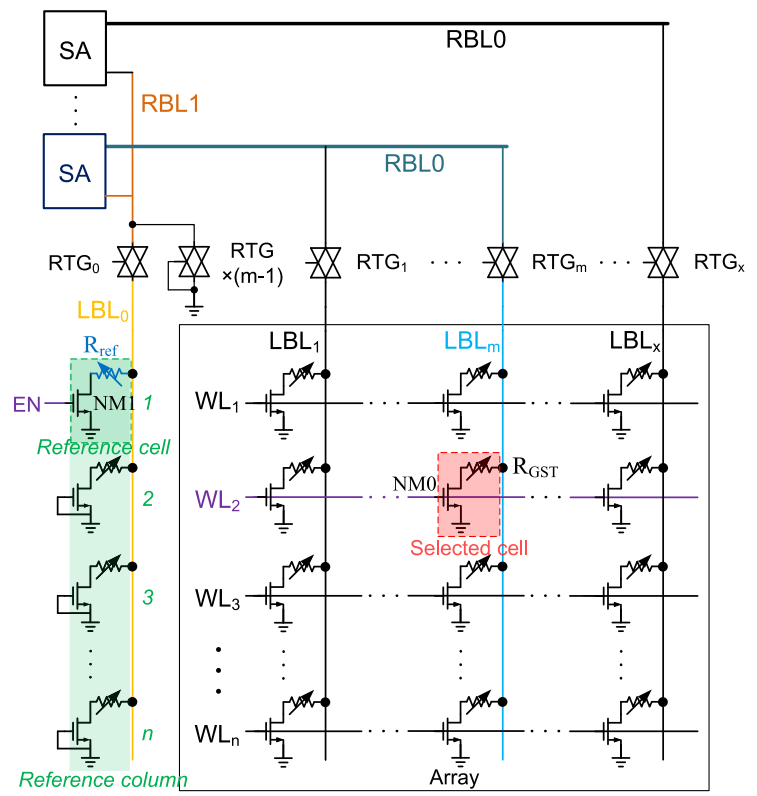

Fig. 3. Proposed read method.

read current. If a constant reference current is still being used, the read access time will be longer. Fig. 2(a) is the simulation results of conventional read access time. $R_{G S T}$ are $30 \mathrm{~K} \Omega$ (set) and $2 \mathrm{M} \Omega$ (reset), respectively. The read access time is $221.6 \mathrm{~ns}$, which is determined by reset speed. Fig. 2(b) shows the relationship between $I_{\text {read }}$ and $I_{\text {ref }}$ versus time. At first, set state $I_{\text {read }}$ and reset state $I_{\text {read }}$ rise to their maximum currents. Then they begin to drop as the natural exponential curves, just like the charging process of a capacitor. Due to their resistances difference, set state $I_{\text {read }}$ is higher than reset state $I_{\text {read }}$ when they reach a stable value. In the conventional method, $I_{r e f}$ is independent of time. When reading a reset selected cell, DO will not output a correct result until reset state $I_{\text {read }}$ is lower than $I_{\text {ref }}$. A pseudo reading zone is therefore created.

\section{Proposed read method}

In the read operation, the extra charging process of parasitic capacitors causes a long readout time. If $I_{\text {ref }}$ is designed to be changeable like $I_{\text {read }}$, the read access time may be reduced. Proposed read method is shown in Fig. 3. A new reference column which comprises one reference cell and $n-1$ dummy cells is introduced. The reference cell comprises a reference poly-resistance $\mathrm{R}_{\text {ref }}$ and a NMOS transistor NM1. $R_{\text {ref }}$ is set between the highest resistance in set distributions and the lowest resistance in reset distributions. Gate of NM1 is controlled by EN signal. Dummy cell is composed of a GST element and a NMOS transistor. The gates of NMOS are connected to the ground, so these NMOS are always switched off. The reference cell and the selected cell have the same bit line parasitic parameters. Read transmission gate $\mathrm{RTG}_{0}$ controls the read operation. Besides, an extra RTG is connected between RBL1 (Read Bit Line) and the ground. This RTG has the same topology and size as RTGs in the array. Multiplier of the extra RTG is $m-1$. Control terminal of the extra RTG is connected to ground, so this RTG is always switched off. In this way, the reference cell and the selected cell have the same RTG parasitic 
parameters. $\mathrm{LBL}_{0}$ is connected to RBL1 through $\mathrm{RTG}_{0}$. RBL0 and RBL1 are connected to SAs. During the read operation, with the help of the selected EN, WL and RTGs, two cells are connected to the SA: a reference cell through RBL1, a cell to be measured through RBL0. In the reference side, there are one selected cell, $n-1$ unselected cells, one selected RTG and $m-1$ unselected RTGs; in the array, there are also one selected cell, $n-1$ unselected cells, one selected RTG and $m-1$ unselected RTGs. Different from the conventional methods which use a constant reference current $[6,7]$, the reference cell and the selected cell have the same parasitic elements in the proposed method.

Fig. 4(a) shows schematic of the proposed SA. Compared to the aforementioned method, NM6 and a new reference current $I_{\text {refnew }}$ are introduced. NM6 helps $V_{\text {clamp }}$ generate $I_{\text {refnew }}$ from the reference cell. Then $I_{\text {refnew }}$ is compared with $I_{\text {read }}$. Fig. 4(b) shows schematic of the $V_{\text {clamp }}$ generator. $V_{b g}$ is the bandgap voltage. The proposed method uses one voltage ( $\left.V_{\text {clamp }}\right)$ to generate $I_{\text {refnew }}$ and $I_{\text {read }}$, which is different from previous methods which use two different voltages/circuits to generate $I_{\text {read }}$ and $I_{\text {ref }}[6,7,8]$. In the proposed method, $I_{\text {refnew }}$ and $I_{\text {read }}$ are more likely to have a similar variation trend. The design is symmetric in terms of parasitic parameters and SA topology. A short current signal instead of a long current signal routing from the SA to reference cells is used [8].

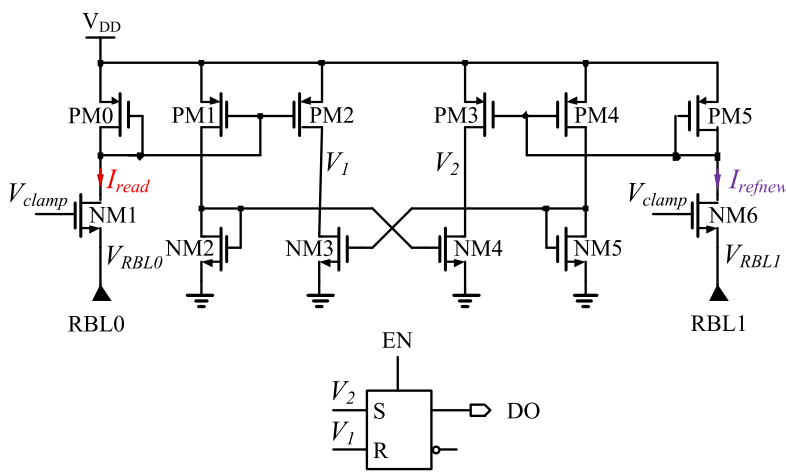

(a)

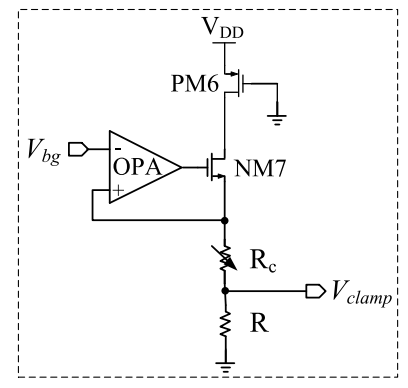

(b)

Fig. 4. (a) The schematic of the proposed SA. (b) Generator of the clamp voltage $V_{\text {clamp }}$.

In many non-volatile memories, the reference current is generated by averaging the read-out current of paired high resistance state (HRS) and low resistance state (LRS) written reference cells [8,9]. But large deviations of HRS and LRS will cause reference-currents deviation. Therefore, the single-reference scheme has wider sense margin if properly designed. Compared to the previous method $[8,9,10]$, no reference cells need to be programmed in the initialization phase.

\section{Performance evaluation}

Simulated in SMIC $40 \mathrm{~nm}$ CMOS process, the proposed method is utilized in 4-Mb PCM to verify performance. The 4-Mb PCM is used to replace SRAM and EEPROM in applications like printer and Bluetooth speaker. In the array and the reference column, we have $\mathrm{n}=1024, \mathrm{~m}=64$. Fig. 5(a) and Fig. 5(b) are the 
simulation results of readout time when $\mathrm{WL}_{1}$ and $\mathrm{WL}_{1024}$ are selected, respectively. $R_{\text {ref }}$ is $300 \mathrm{~K} \Omega$. $R_{G S T}$ are $30 \mathrm{~K} \Omega$ (set) and $2 \mathrm{M} \Omega$ (reset), respectively. In the simulation, parasitic resistances and capacitances of the bit line metal are also taken into account. Parasitic resistance of the whole LBL is about $68.6 \Omega$ and parasitic capacitance of the whole LBL is about $14 \mathrm{fF}$. Parasitic capacitance of the GST element is considered and it is approximately $18.86 \mathrm{aF}$. The read access time is $30.65 \mathrm{~ns}$, which is determined by set speed of Fig. 5(b).

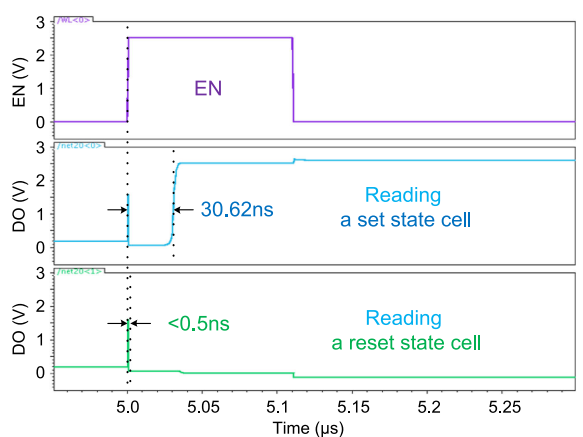

(a)

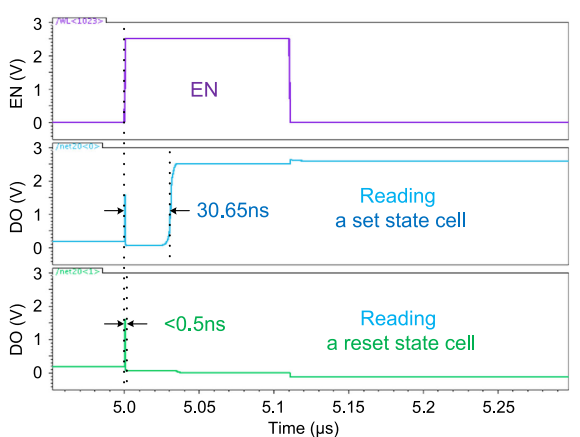

(b)

Fig. 5. (a) The simulation result of readout time when $\mathrm{WL}_{1}$ is selected. (b) The simulation result of readout time when $\mathrm{WL}_{1024}$ is selected.

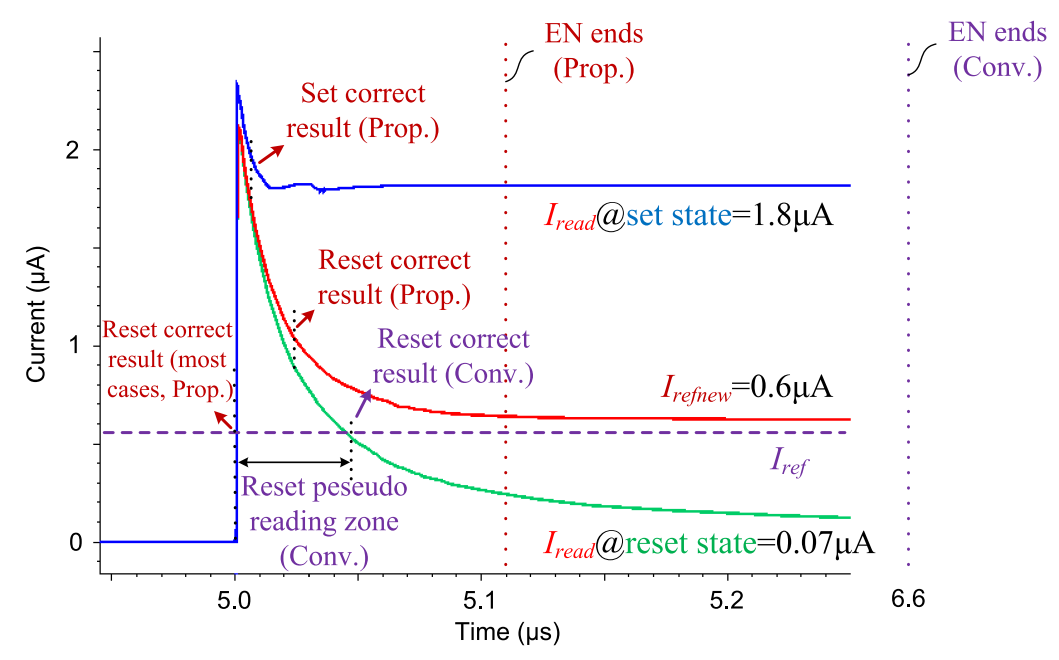

Fig. 6. Currents comparison in the proposed method: Conv.: conventional method; Prop.: proposed method.

Fig. 6 shows the relationship between $I_{\text {read }}$ and $I_{\text {refnew }}$ versus time. In this new scheme, $I_{\text {refnew }}$ first rise to its maximum current, then begin to drop as the natural exponential curve, finally reach a stable value. $I_{\text {refnew }}$ curve has been in the middle of set state $I_{\text {read }}$ and reset state $I_{\text {read }}$ early, since the reference cell and the selected cell have the same parasitic parameters. When reading a set selected cell: as $I_{\text {refnew }}$ is lower than $I_{\text {read }}$, DO will output a correct result. When reading a reset selected cell: as $I_{\text {refnew }}$ is higher than $I_{\text {read }}$, DO will output a correct result. When $I_{\text {refnew }}$ and $I_{\text {read }}$ are the same, $V_{1}$ and $V_{2}$ are the same, and SR latch output " 0 ". This is why in most cases DO can always output the correct reset result. The read current is as 
small as $1.8 \mu \mathrm{A}$, which is the smallest read current of PCM in the world, to our best knowledge.

According to the simulation results below, the disable of EN is set above $5.11 \mu \mathrm{s}$ to ensure all the bits are correctly read. On the contrary, conventional disable of $\mathrm{EN}$ is set at $6.6 \mu \mathrm{s}$.

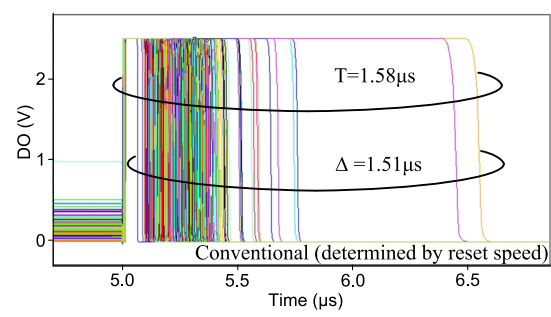

(a)

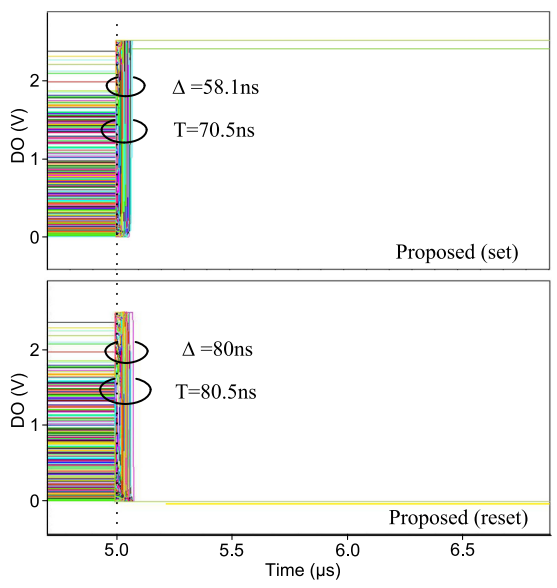

(b)

Fig. 7. (a) Monte Carlo simulation result of the conventional method. (b) Monte Carlo simulation results of the proposed method.

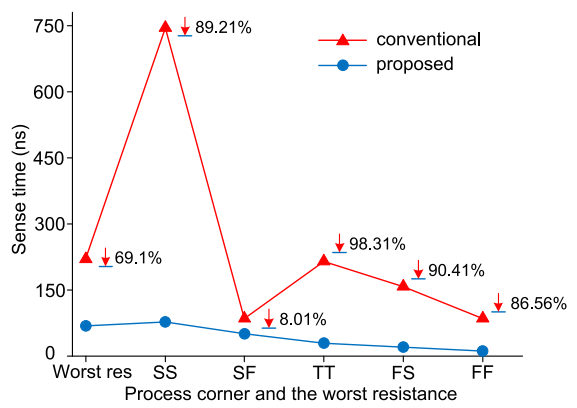

Fig. 8. Comparisons of the read access time under different process corners and the worst $R_{G S T}$.

Monte Carlo simulation results of the read access time are shown in Fig. 7. It can be used to compare the worst case of the conventional and proposed method. Monte Carlo simulations are performed using the industry compatible SMIC 40-nm model parameters. Accomplished in process \& mismatch analysis, three times standard deviation $(3 \sigma)$ is used as the variances of parameters and mismatch of MOS and resistor. The GST device is modeled as the resistance distribution in order to reflect the effect of process variation [6]. In each simulation, 4000 trials were run. Variations of $V_{\text {clamp }}$, parasitic capacitance of the GST element and parasitic elements of the bit line metal are also considered. Circuits in Fig. 3, Fig. 4(a) and Fig. 4(b) are all included in Monte Carlo simulation. The worst read access time of the proposed method (determined by set speed) is $80.5 \mathrm{~ns}$. On the contrary, the worst read access time of the conventional method (determined by reset speed) is $1.58 \mu \mathrm{s}$. It is clearly that the worst read access time is largely reduced. Timing 
margin between the regular case and the worst case is reduced from $1.51 \mu$ s to $80 \mathrm{~ns}$.

Fig. 8 shows the comparisons of the read access time with the above two methods under different process corners and the worst $R_{G S T}$, respectively. The worst $R_{G S T}$ is $100 \mathrm{~K} \Omega$ (set) and $1 \mathrm{M} \Omega$ (reset), respectively.

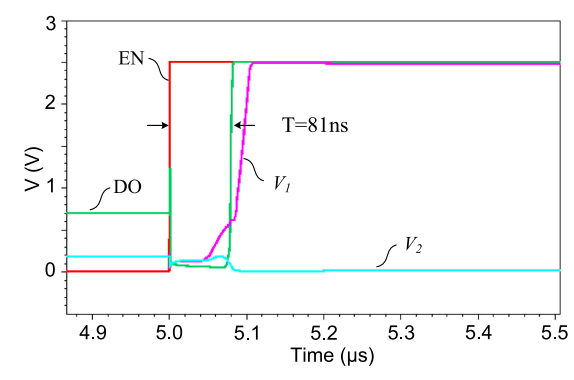

(a)

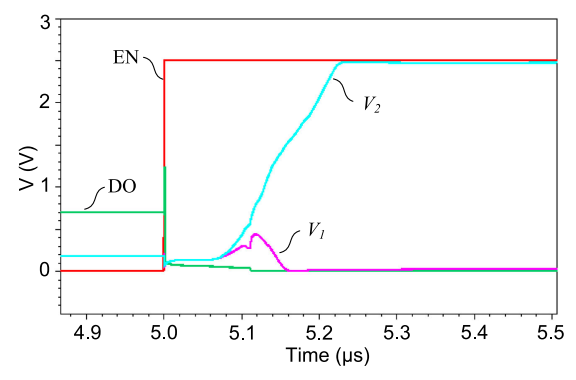

(b)

Fig. 9. The read operation under both the process corner SS and the worst $R_{G S T}$ : (a) reading a set cell; (b) reading a reset cell.

Fig. 9 is the simulation results of the read operation under both the process corner SS and the worst $R_{G S T}$. When SA begins to work, $V_{1}$ and $V_{2}$ are charged and begin to rise; the read current and the reference current begin to charge parasitic elements. As $I_{\text {read }}$ and $I_{\text {refnew }}$ are almost the same in this moment, $V_{1}$ and $V_{2}$ are the same. When $V_{1}$ and $V_{2}$ are the same, SR latch outputs " 0 ". When reading a set cell, after distinguishing point of the proposed method, $V_{1}$ is nearly $2.5 \mathrm{~V} ; V_{2}$ is nearly $0 \mathrm{~V}$. SR latch outputs " 1 ". When reading a reset cell, after charging process, $V_{1}$ is nearly $0 \mathrm{~V} ; V_{2}$ is nearly $2.5 \mathrm{~V}$. SR latch outputs " 0 ". The worst variation of process corner and $R_{G S T}$ won't influence a correct readout result.

Fig. 10 and Fig. 11 are the simulation results of the read operation under both the worst variation of $\mathrm{V}_{\mathrm{TH}}$ and $R_{G S T}$. Increase or decrease of $\mathrm{V}_{\mathrm{TH}}$ of the transistors in the SA are marked by arrows in Fig. 10(a) and Fig. 11(a). Variation of $\mathrm{V}_{\mathrm{TH}}$ is about $70 \mathrm{mV}$, which is the worst variation of $\mathrm{V}_{\mathrm{TH}}$ in SMIC $40 \mathrm{~nm}$ CMOS process. In Fig. 10, the selected cell is a set cell. $R_{G S T}$ is $100 \mathrm{~K} \Omega$. The variations of $\mathrm{V}_{\mathrm{TH}}$ make $I_{\text {read }}$ decreases and $I_{\text {refnew }}$ increases. This results in a long pseudo reading zone in Fig. 10(b). In Fig. 11, the selected cell is a reset cell. $R_{G S T}$ is $1 \mathrm{M} \Omega$. The

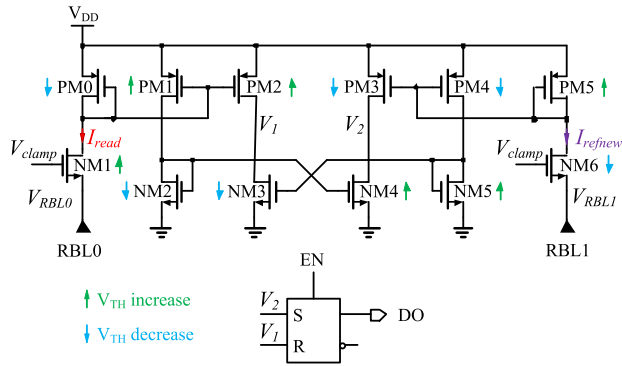

(a)

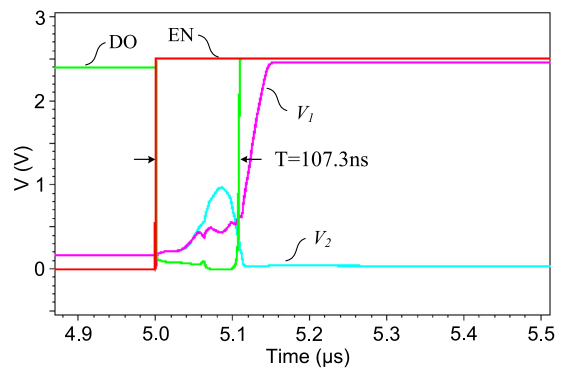

(b) 
variations of $\mathrm{V}_{\mathrm{TH}}$ make $I_{\text {read }}$ increases and $I_{\text {refnew }}$ decreases. The worst variation of $\mathrm{V}_{\mathrm{TH}}$ and $R_{G S T}$ won't influence a correct readout result.

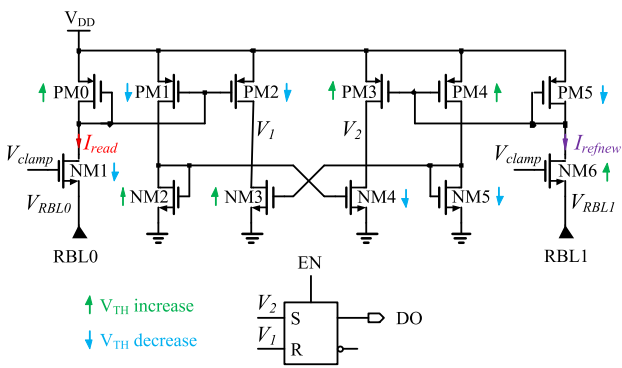

(a)

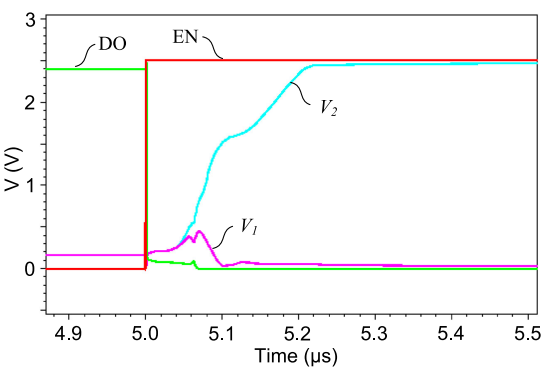

(b)

Fig. 11. When the selected cell is a reset cell: (a) variations of $\mathrm{V}_{\mathrm{TH}}$; (b) the simulation result.

The comparison between the proposed read method and the prior read methods is shown in Table I. In the read circuit of memory, the read access time will decrease as bits per SA (the number of cells sharing a SA) decrease or the read current increases. In Table I, we try to compare their performance in a same standard.

Table I. Performance summary and comparison

\begin{tabular}{l|l|l|l|l|l}
\hline & This paper & Ref. [7] & Ref. [11] & Ref. [12] & Ref. [13] \\
\hline Technology & $\begin{array}{l}40 \mathrm{~nm} \\
\text { PCM }\end{array}$ & $\begin{array}{l}130 \mathrm{~nm} \\
\text { PCM }\end{array}$ & $\begin{array}{l}90 \mathrm{~nm} \\
\text { PCM }\end{array}$ & $\begin{array}{l}90 \mathrm{~nm} \\
\text { PCM }\end{array}$ & $\begin{array}{l}130 \mathrm{~nm} \\
\text { PCM }\end{array}$ \\
\hline Power supply $(\mathrm{V})$ & 2.5 & 1.5 & 1.8 & 1.2 & 3.3 \\
\hline Read access time $T(\mathrm{~ns})$ & 30.65 & 9.9 & 37.5 & 12 & 98 \\
\hline Bits per SA $F($ Kbit) & 64 & 8 & 4 & 32 & 0.125 \\
\hline $\begin{array}{l}\text { Cell read current (set) } I_{s} \\
(\mu \mathrm{A})\end{array}$ & 1.8 & 20 & N/A & 55 & 18 \\
\hline$T / F(\mathrm{~ns} / \mathrm{Kb})$ & 0.48 & 1.24 & 9.375 & 0.38 & 784 \\
\hline$T \cdot I_{S}(\mathrm{~ns} \cdot \mu \mathrm{A})$ & 55.26 & 198 & N/A & 660 & 1764 \\
\hline$T \cdot I_{s} / F(\mathrm{~ns} \cdot \mu \mathrm{A} / \mathrm{Kb})$ & 0.86 & 24.75 & N/A & 20.63 & 14112 \\
\hline
\end{tabular}

\section{Conclusions}

Parasitic elements on BL have always been a big limit for the read performance in various kinds of memories. This paper presents a novel read method for 4-Mb PCM in $40 \mathrm{~nm}$ CMOS process. The proposed method uses a reference column to generate a reference current. The reference column is composed of one reference cell and a certain number of $1 \mathrm{~T} 1 \mathrm{R}$ cells. The reference cell and the selected array cell have the same parasitic parameters in the read operation. Simulation results show a $30.65 \mathrm{~ns}$ read access time compared to the previous $221.6 \mathrm{~ns}$. Monte Carlo simulation shows a $80.5 \mathrm{~ns}$ worst read access time compared to the conventional $1.58 \mu \mathrm{s}$. This method can be easily applied in the practical products and it is also useful in MRAM, RRAM and Flash. 


\section{Acknowledgments}

This work was supported by the "Strategic Priority Research Program" of the Chinese Academy of Sciences (XDA09020402), National Key Basic Research Program of China (2013CBA01900), National Integrate Circuit Research Program of China (2009ZX02023-003). 